Doi: $10.15863 /$ TAS

\section{International Scientific Journal Theoretical \& Applied Science}

p-ISSN: 2308-4944 (print) e-ISSN: 2409-0085 (online)

Year: $2014 \quad$ Issue: $11 \quad$ Volume: 19

Published: $30.11 .2014 \quad$ http://www.T-Science.org

SECTION 2. Applied mathematics. Mathematical modeling.
Vladimir Nikolayevich Kestelman

Specialty Scientific Consultant, Russian Technology Initiative, LTD, President of KVN International, Inc., Philadelphia, Member of Mid-Atlantic-Russia Business Council and of International Visitors Council of Philadelphia, King of Prussia, Pennsylvania, USA

Abdizhahan Manapovich Sarsenbi Professor, doctor of physico-mathematical Sciences South Kazakhstan state University M.O. Auezova,

Kazakhstan abzhahan@mail.ru

Alexandr Nikolayevich Shevtsov candidate of technical sciences, Member of PILA (USA), department of Mathematics, deputy director on Science of FITAT, Taraz state University M.Kh. Dulati, Kazakhstan Shev_AlexXXXX@mail.ru

\title{
ABOUT ONE FAST-CHANGING CHARACTERISTIC EQUATION SPECTRAL PROBLEMS FOR DIFFERENTIAL EQUATIONS OF SECOND ORDER
}

\footnotetext{
Abstract: The paper discusses some of the solutions of the characteristic equation of the spectral problem. Solutions obtained by numerical methods.

Key words: spectral problem, the beam, the basicity of the operator, linearization, Maple.

Language: Russian

Citation: Kestelman VN, Sarsenbi AM, Shevtsov AN (2014) ABOUT ONE FAST-CHANGING CHARACTERISTIC EQUATION SPECTRAL PROBLEMS FOR DIFFERENTIAL EQUATIONS OF SECOND ORDER. ISJ Theoretical \& Applied Science 11 (19): 1-6. doi: http://dx.doi.org/10.15863/TAS.2014.11.19.1

\section{ОБ ОДНОМ БЫСТРО МЕНЯЮЩЕМСЯ РЕШЕНИИ ХАРАКТЕРИСТИЧЕСКОГО УРАВНЕНИЯ} СПЕКТРАЛЬНОЙ ЗАДАЧИ ДЛЯ ДИФФЕРЕНЦИАЛЬНОГО УРАВНЕНИЯ ВТОРОГО ПОРЯДКА

Аннотация: В работе рассматриваются некоторые решения характеристического уравнения спектральной задачи. Решения получены численными методами.

Ключевые слова: спектральная задача, пучок, базисность, оператор, линеаризация, Maple.
}

Многочисленные исследования свойств собственных функций пучков операторов показывают, что операторные пучки трудно поддаются изучению [1]. Поэтому при изучении конкретных задач исследователи часто изучают подсистему собственных функций пучков, а также базисность подсистемы собственных функций пучков различного порядка с краевыми условиями типа Дирихле.

Изучению свойств собственных функций пучков операторов посвящено много работ. Одним из основных методов изучения свойств собственных функций операторных пучков является так называемый, метод линеаризаций, предложенный М.В. Келдышем [2]. В монографиях А.С. Маркуса [4], И.М. Гохберга и
М.Г. Крейна [3] развиваются различные способы линеаризации операторных пучков. Можно сказать, что основное содержание монографии А.С. Маркуса [4] посвящена теории не глобальной линеризации пучка, а такой частичной линеризации, которая эффективна при рассмотрении определенной части спектра. Этот способ основан на нахождении, так называемого, спектрального корня пучка.

В работе М.Г. Джавадова [9] показана полнота в $L_{2}(0,1)$ половины собственных функции пучка второго порядка

$$
y^{\prime \prime}(x)+2 B \lambda y^{\prime}(x)+C \lambda^{2} y(x)=0,
$$




$$
\begin{aligned}
& y^{\prime \prime}(0)+a \lambda y(0)=0, \\
& y^{\prime \prime}(1)+a \lambda y(1)=0,
\end{aligned}
$$

где $C-B^{2}>0$.

Работа В.А. Ильина [5] посвящена вопросам базисности подсистемы собственных функций пучка М. В. Келдыша.

Доказательство того, что система $\left\{u_{k_{1}}, u_{k_{2}}\right\}$ образует полную ортонормированную систему в $L_{2}(1,1)$, т.е. базис Рисса, полностью приведено в [1].

Для исследования периодической задачи рассмотрим спектральную задачу с периодическими краевыми условиями.

Ранее мы рассмотрели пучок с краевыми условиями типа Дирихле. Дальнейшее исследование предполагает изучение пучка четвертого порядка с краевыми условиями периодического типа, антипериодического типа, а также с краевыми условиями типа Штурма. Если половина краевых условий задана на одном конце, а половина краевых условий задана на другом конце рассматриваемого отрезка, то краевые условия такого типа называют краевыми условиями типа Штурма. Во всех случаях мы используем уже изложенную методику исследования свойств подсистемы собственных функций пучка четвертого порядка [1]. Используемая методика достаточно проста, благодаря возможности исследования спектральных задач для дифференциального оператора второго порядка с инволюцией.

Прежде чем приступать к изучению пучка

$$
\begin{gathered}
A=\left(\alpha^{2}-1\right) u^{I V}(x)-2 \alpha \lambda^{2} u^{\prime \prime}(x)+\lambda^{4} u(x), \\
-1<x<1,
\end{gathered}
$$

с краевыми условиями

$$
\begin{gathered}
u(-1)=u(1), \quad u^{\prime}(-1)=u^{\prime}(1), \quad u^{\prime \prime}(-1) \\
=u^{\prime \prime}(1), u^{\prime \prime}(-1)=u^{\prime \prime \prime}(1)
\end{gathered}
$$

рассмотрим спектральную задачу с периодическими краевыми условиями

$$
\begin{gathered}
-u^{\prime \prime}(-x)+\alpha u^{\prime \prime}(x)=\lambda u(x), \quad-1<x<1, \\
u(-1)=u(1), \quad u^{\prime}(-1)=u^{\prime}(1) .
\end{gathered}
$$

Общее решение уравнения (1) имеет вид

$$
u(x)=\operatorname{acos} \sqrt{\frac{\lambda}{1-\alpha}} x+b \sin \sqrt{\frac{\lambda}{-1-\alpha}} x .
$$

Полученные серии частных значений приведены в [1]. Нас же интересует более общий случай и наличие решений уравнения (3).

Решения будем определять в Maple:

$$
\begin{aligned}
& \text { restart; } \\
& g 1:=\operatorname{sqrt}\left(\frac{\text { lambda }}{1-a}\right): \\
& g 2:=\operatorname{sqrt}\left(\frac{- \text { lambda }}{1+a}\right): \\
& R 0:=\frac{\tan (g 1)}{\cot (g 2)}=-\frac{g 2}{g 1} ; \\
& R 00:=\frac{g 1 \cdot \tan (g 1)}{1}=-\frac{g 2}{\tan (g 2)} ;
\end{aligned}
$$$$
R 000:=\operatorname{lhs}(R 00)-r h s(R 00) ;
$$

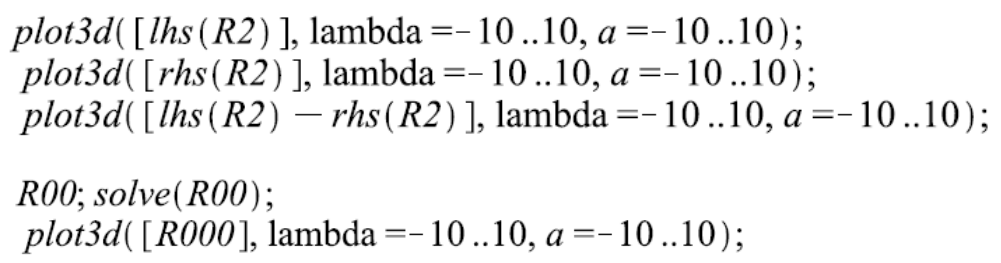
$\operatorname{plot} 3 d([\operatorname{rhs}(R 2)]$, lambda $=-10 . .10, a=-10 . .10)$; plot3d $([\operatorname{lhs}(R 2)-\operatorname{rhs}(R 2)]$, lambda $=-10 . .10, a=-10 . .10)$;$$
\operatorname{plot} 3 d([R 000], \text { lambda }=-10 . .10, a=-10 . .10) \text {; }
$$ 

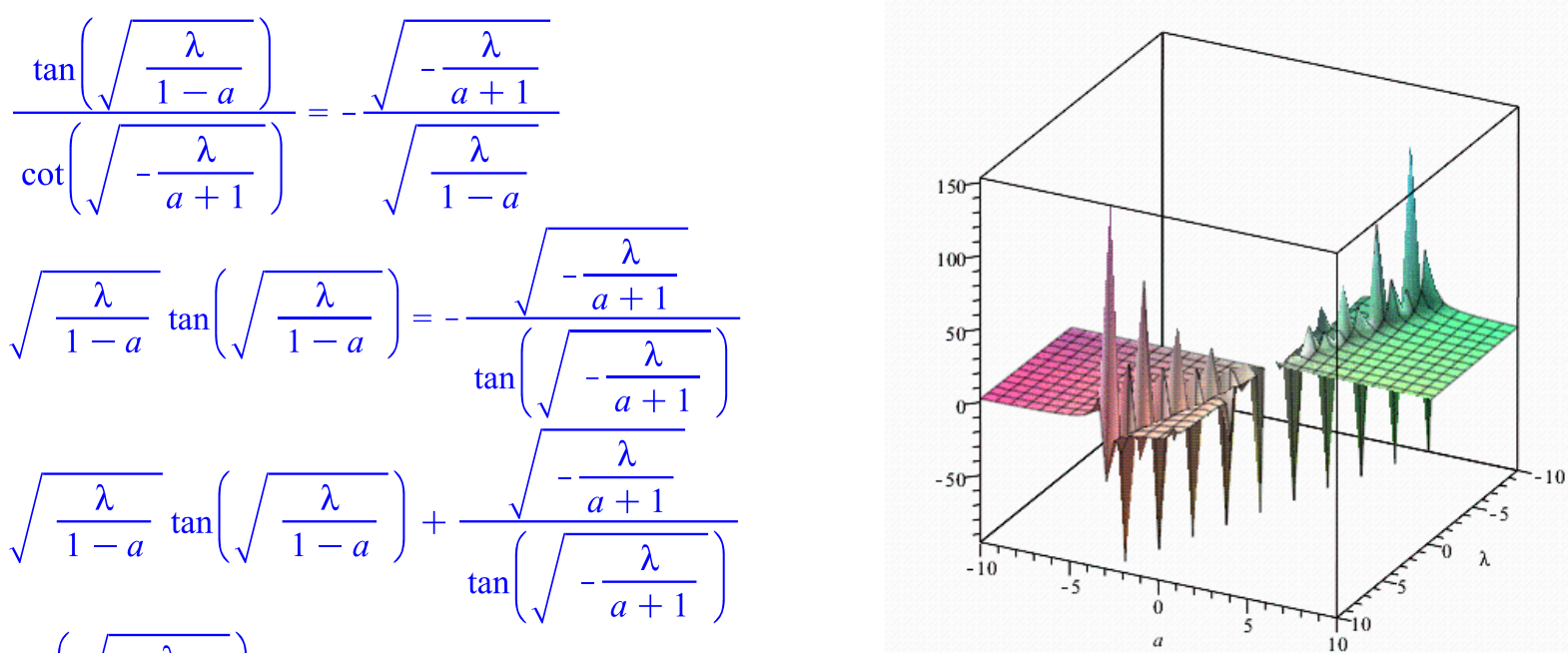

$\tan \left(\sqrt{\frac{\lambda}{1-a}}\right)=-1$
$\cot \left(\sqrt{-\frac{\lambda}{a+1}}\right)=-\frac{\sqrt{-\frac{\lambda}{a+1}}}{\sqrt{\frac{\lambda}{1-a}}}$
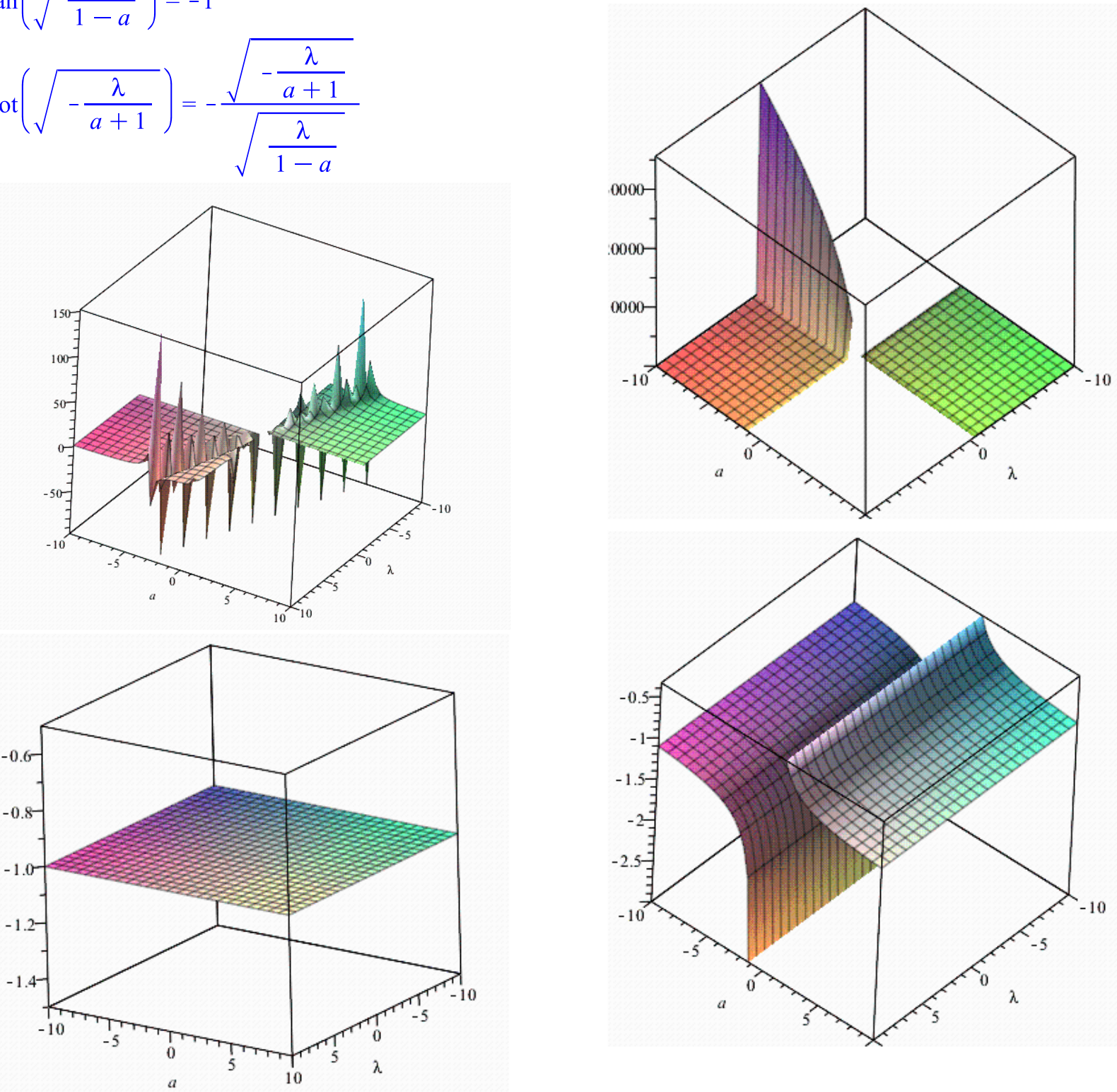

Рисунок 1 - Некоторые промежуточные данные. 
Рассмотрим теперь, после несложных преобразований формулу (3) в следующем виде:

$\sqrt{\frac{\lambda}{1-a}} \tan \left(\sqrt{\frac{\lambda}{1-a}}\right)=-\frac{\sqrt{-\frac{\lambda}{a+1}}}{\tan \left(\sqrt{-\frac{\lambda}{a+1}}\right)}$

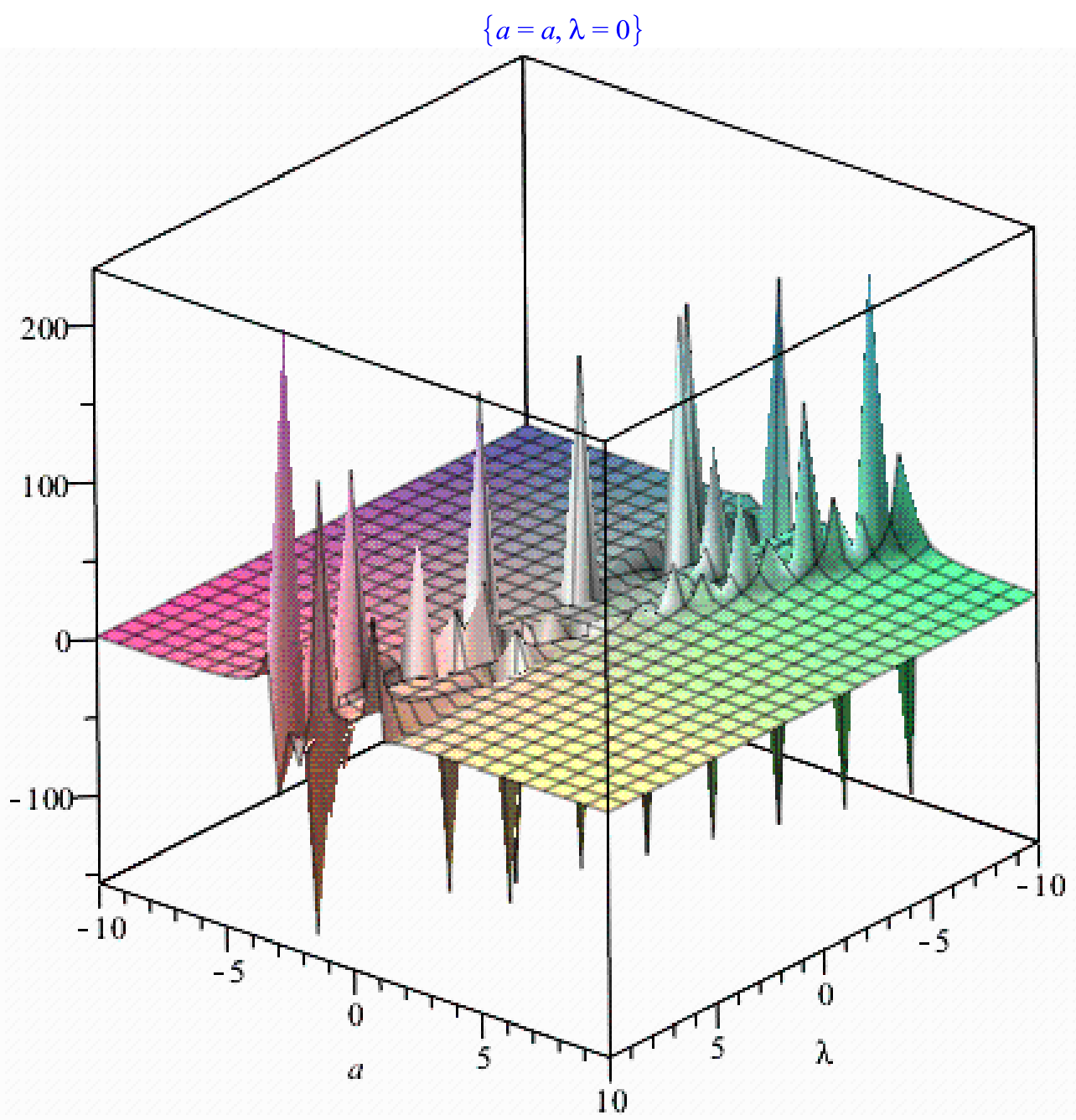

Рисунок 2 - Решение характеристического уравнения спектральной задачи.

for $i$ from -50 to -11 do

$\operatorname{plot}\left(\operatorname{subs}\left(a=\frac{i}{10}, R 000\right)\right.$, lambda $=-20 . .20$, title $=$ evalf $\left(\frac{i}{10}\right)$, color $=$ red $)$;

od;

for $i$ from -9 to 9 do

$\operatorname{plot}\left(\operatorname{subs}\left(a=\frac{i}{10}, R 000\right)\right.$, lambda $=-20 . .20$, title $=$ evalf $\left(\frac{i}{10}\right)$, color $=$ red $)$;

od;

for $i$ from 11 to 50 do

$\operatorname{plot}\left(\operatorname{subs}\left(a=\frac{i}{10}, R 000\right)\right.$, lambda $=-20 . .20$, title $=$ evalf $\left(\frac{i}{10}\right)$, color $=$ red $)$;

od; 

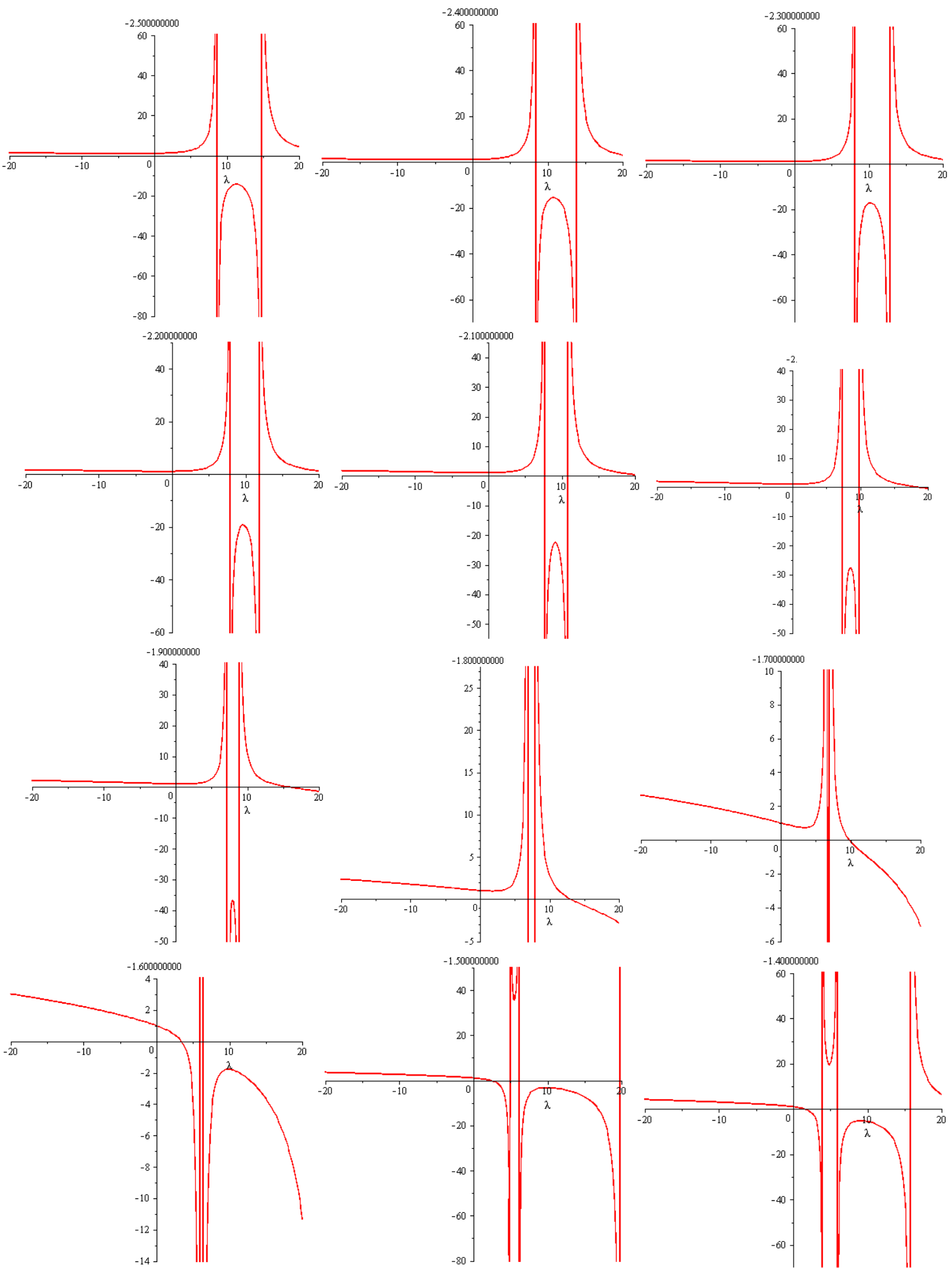

Рисунок 3 - Некоторые из частных решений характеристического уравнения спектральной задачи. 


\section{Выводы.}

При любых значениях $\alpha$ спектральная задача (7), (8) имеет собственные функции

$$
\begin{gathered}
u_{k_{1}}(x)=\sin \left(\frac{\pi}{2}+k \pi\right) x \\
u_{k_{2}}(x)=\cos k \pi x
\end{gathered}
$$

Полученные численные данные подтверждают существование нетривиальных решений.
Выписанные нами системы собственных функций совпадают с подсистемами собственных функции пучка (1),(2). Факт базисности Рисса выписанных систем является следствием полноты, ортогональности и почти нормированности систем собственных функций спектральной задачи. В свою очередь, последние являются следствиям самосопряженности спектральной задачи.

Получен ряд нетривиальных решений на промежутке $\lambda=\overline{-20,20}$, при $a=\overline{-2.1,5.0}$ с шагом $h=0.1$.

\section{References:}

1. Sarsenbi AM (2014) Research report IMM 09.11. 2014.

2. Keldysh MV (1971) O polnote sobstvennykh funktsiy nekotorykh klassov nesamosopryazhennykh lineynykh operatorov. Uspekhi matematicheskikh nauk. T. 26, No.4 (160). - pp. 15-41.

3. Gokhberg, IT, Kreyn MG (1965) Vvedenie v teoriyu lineynykh nesamosopryazhennykh operatorov $\mathrm{v}$ gil'bertovom prostranstve: monografiya. Moscow: Nauka, 448.

4. Markus AS (1986) Vvedenie v spektral'nuyu teoriyu polinomial'nykh operatornykh puchkov:monografiya: - Kishinev: Shtinitsa, 260.

5. Il'in VA (1976) Neobkhodimye i dostatochnye usloviya bazisnosti podsistemy sobstvennykh i prisoedinennykh funktsiy puchka M.V. Keldysha obyknovennykh differentsial'nykh operatorov. Dokl. AN SSSR. 1976. -T.227, No. 4, pp. 28-31.

6. Shkalikov AA (1983) Kraevye zadachi dlya obyknovennykh differentsial'nykh uravneniy s parametrom v granichnykh usloviyakh. Trudy sem. im. I.G.Petrovskogo. No. 9, pp. 190-229.

7. Vagabov AI (1994) Vvedenie v spektral'nuyu teoriyu operatorov:monografiya.- Rostov n/Donu: Izdvo Rostovskogo universiteta.

8. Vagabov AI (2012) Ob usloviyakh kratnoy razlozhimosti funktsiy po kornevym elementam puchka obyknovennykh differentsial'nykh operatorov. Differentsial'nye uravneniya. 2012. - T. 48. No.8, pp. 1067-1079.

9. Dzhavadov MG (1964) O polnote nekotoroy chasti sobstvennykh funktsiy nesamosopryazhennogo differentsial'nogo operatora. DAN SSSR. T.159, No. 4, pp. 723725 .

10. Stepin SA (1993) Spektr i polnota sobstvennykh kolebaniy atmosfery $\mathrm{s}$ temperaturnoy stratifikatsiey. Matematicheskiy sbornik. T.184, No.6, pp. 83-98.

11. Sarsenbi AM (2010) Bezuslovnye bazisy, svyazannye s neklassicheskim differentsial'nym operatorom vtorogo poryadka. Differentsial'nye uravneniya. T.46, No.4, pp. 506-511.

12. Sarsenbi AM, Tengaeva AA (2012) O bazisnykh svoystvakh kornevykh funktsiy dvukh obobshchennykh spektral'nykh zadach. Differentsial'nye uravneniya. T.48, No.2, pp.294-299. 